UT-13-04

\title{
Non-Thermal Production of Wino Dark Matter via the Decay of Long-Lived Particles
}

\author{
Takeo Moroi, Minoru Nagai and Masahiro Takimoto \\ Department of Physics, University of Tokyo, Tokyo 113-0033, Japan
}

\begin{abstract}
We consider supersymmetric models in which the neutral Wino is the lightest superparticle (LSP), and study the possibility that non-thermally produced Wino plays the role of dark matter. The thermal relic density of Wino is smaller than the present mass density of dark matter if $m_{\tilde{W}} \lesssim 2.9 \mathrm{TeV}$; however, even with smaller Wino mass, the Wino can be the dominant component of dark matter if it is non-thermally produced by the decay of a long-lived particle. In order to study such a possibility in detail, we perform a precise calculation of the present mass density of Wino produced by the decay of a long-lived particle, taking account of the following effects: (i) coannihilation among charged and neutral Winos, and (ii) Sommerfeld effect on the pair annihilation cross section of Winos. We consider several well-motivated cases where the long-lived particle corresponds to cosmological moduli fields, gravitino, or axino, and discuss the implication of the Wino LSP for these cases.
\end{abstract}




\section{Introduction}

In models with low-energy supersymmetry (SUSY), there may exist various long-lived particles (which we call $X$ ) which have very weak interactions with the particles in the minimal SUSY standard model (MSSM). The examples include moduli fields, Polonyi field (responsible for SUSY breaking), gravitino (which is the superpartner of graviton), axino (which is the superpartner of axion in SUSY Peccei-Quinn model), and so on. In most of the cases, these particles are irrelevant for low-energy phenomenology because of the weakness of their interactions.

Cosmologically, however, they often cause serious problems [1, 2, 3, 4, 5] because, even though their interactions are very weak, a sizable amount of them may be produced in the early universe. If they are stable, they survive until today, resulting in the overclosure of the universe. Even if they are unstable, their lifetimes are often so long that they decay at a very late stage of the evolution of the universe. If they decay after the big-bang nucleosynthesis $(\mathrm{BBN})$ starts, hadro- and photo-dissociation processes of light elements induced by their decay products may spoil the success of the BBN scenario. In order to avoid such a problem, the lifetime of $X$ is required to be shorter than $\sim 1$ sec; otherwise, the abundance of $X$ is bounded above [6, 7].

Even with lifetime shorter than $\sim 1$ sec, $X$ may cause other cosmological difficulties. In our study, we consider one of such problems: the overproduction of the lightest superparticle (LSP) due to its decay. In many cases, $X$ decays into superparticles which cascade down to the LSP. Then, if all the produced LSPs survive until today, it is often the case that the resultant mass density of the LSP becomes much larger than the present energy density of dark matter. In particular, the universe may be once dominated by $X$ before the decay of $X$; in such a case, the overproduction of the LSP is a serious problem.

If the pair annihilation cross section of the LSP is large enough, the LSPs produced by the decay of $X$ may annihilate and the abundance of the LSP can be suppressed. There exists a viable and well-motivated candidate of such LSP, the neutral Wino $\tilde{W}^{0}$. In models with the naive grand-unified-theory (GUT) relation among gaugino masses, the Wino cannot be the LSP. However, even if the unification of the gauge group is realized, the GUT relation can be easily violated in various cases, for example, in the anomaly-mediation model [8, 9], product-group unification [10], and so on. In particular, in the light of the recent discovery of the Higgs-like particle at the LHC [11, 12], the anomaly-mediation model with large scalar masses [8] (as large as $\sim 10-100 \mathrm{TeV}$ ) is well-motivated because the relatively large SUSY Higgs mass of $\sim 126 \mathrm{GeV}$ can be realized if the scalar tops are so heavy [13]. It is well-known that the Wino can naturally be the LSP in anomaly-mediation model.

In this paper, we reconsider the possibility of the non-thermally produced Wino being dark matter. In particular, we precisely calculate the relic abundance of the neutral Wino. The possibility of Wino dark matter from the decay of long-lived particles was first discussed in [8, 14], in which it was shown that the relic density of the Wino can be consistent with the dark matter density. Then, such a scenario has been applied to various cases [15, 16, 17, 18, 19]. (For the case where the LSP is not Wino-like, see also [20, 21, 22, 23.) Compared 
to the previous studies, we have carefully taken into account the following in the calculation of the relic Wino abundance:

(i) Coannihilation effect among neutral and charged Winos, which becomes important when the decay temperature of $X$ is higher than the mass difference between charged and neutral Winos.

(ii) Sommerfeld enhancement of the pair annihilation cross section, which is large when the Wino is heavier than $\sim 1 \mathrm{TeV}$.

The organization of this paper is as follows. In Section 2, we show relevant formulas to calculate the relic abundance of neutral Wino. In Section 3, we numerically calculate the relic abundance of the Wino for several situations and discuss implications. Section 4 is devoted to conclusions and discussion.

\section{Formulas}

In this section, we summarize the basic formulas to calculate the thermal relic abundance of the neutral Wino through the decay of a heavy particle $X$.

The Wino is $S U(2)_{L}$-triplet, and there exist neutral and charged Winos, denoted as $\tilde{W}^{0}$ and $\tilde{W}^{ \pm}$, respectively. In the present study, we consider the case where the neutral Wino is the LSP; in the following, we assume that superparticles other than Winos are so heavy that they are irrelevant at the time of the freeze-out of Winos. This is the case in the anomalymediated model, which is one of the important motivations of our study. In addition, because we assume that the Higgsinos are much heavier than Winos, the mass difference between $\tilde{W}^{0}$ and $\tilde{W}^{ \pm}$are dominantly from one-loop diagrams with gauge bosons (i.e., $\gamma, Z$, and $W^{ \pm}$) and Winos inside the loop. Then, if the Wino mass is much smaller than the Higgsino mass, the mass difference is typically $150-165 \mathrm{MeV}$ [24, 25], which is insensitive to the Wino mass.

Because the mass difference is much smaller than the Wino mass, the number density of the charged Wino at the time of the freeze-out of Winos may be sizable. Thus, in the calculation of the relic density of $\tilde{W}^{0}$, we include effects of all the possible channels of Wino annihilation. We consider the case where Winos are much lighter than other superparticles, so the coannihilation with those are neglected in the calculation of the relic density.

Now we discuss the evolution of the number density of Wino. As we mentioned, we consider the case with a long-lived particle $X$ which decays into MSSM particles. Using the fact that all the MSSM superparticles eventually decay into charged or neutral Wino, the relevant set of Boltzmann equations to calculate the relic abundance of Wino LSP is given 
by

$$
\begin{aligned}
\frac{d n_{\tilde{W}}}{d t}+3 H n_{\tilde{W}} & =-\left\langle\sigma_{\mathrm{eff}} v\right\rangle\left(n_{\tilde{W}}^{2}-n_{\tilde{W}, \text { eq }}^{2}\right)+N_{\tilde{W}} \Gamma_{X} n_{X}, \\
\frac{d n_{X}}{d t}+3 H n_{X} & =-\Gamma_{X} n_{X}, \\
\frac{d \rho_{\mathrm{rad}}}{d t}\left(1+\frac{1}{3} \frac{\partial \ln g_{*}}{\partial \ln T}\right) & =\left(-4 H \rho_{\mathrm{rad}}+q\right)\left(1+\frac{1}{4} \frac{\partial \ln g_{*}}{\partial \ln T}\right),
\end{aligned}
$$

where $n_{\tilde{W}}$ is the sum of the number densities of neutral and charged Winos, $n_{X}$ is the number density of $X$, and $q$ is a heat injection into radiation as

$$
q=\left(m_{X}-N_{\tilde{W}} m_{\tilde{W}}\right) \Gamma_{X} n_{X}+m_{\tilde{W}}\left\langle\sigma_{\mathrm{eff}} v\right\rangle n_{\tilde{W}}^{2}
$$

with $N_{\tilde{W}}$ being the averaged number of SUSY particles produced by the decay of one $X$. In addition, $\rho_{\text {rad }}$ is the energy density of the relativistic component, and is related to the cosmic temperature $T$ as

$$
\rho_{R}=\frac{\pi^{2}}{30} g_{*}(T) T^{4}
$$

where $g_{*}(T)$ is the effective number of relativistic degrees of freedom.\#1 In our calculation, we approximated that the full particle content at the temperature above the QCD scale (which is taken to be $200 \mathrm{MeV}$ in our analysis) is that of the MSSM, while that at the temperature below the QCD scale consists of photon, three generations of leptons, and pions. Furthermore, $n_{\tilde{W} \text {, eq }}$ denotes the thermal-equilibrium value of $n_{\tilde{W}}, H$ is the expansion rate of the universe, $\Gamma_{X}$ is the decay rate of $X$, and $m_{X}$ and $m_{\tilde{W}}$ are the masses of $X$ and Wino, respectively \#2 In the above Boltzmann equations, the thermally-averaged effective annihilation cross section $\left\langle\sigma_{\text {eff }} v\right\rangle$ accounts both for the coannihilation effect and the Sommerfeld effect, which were not fully taken into accounts in previous analyses.

The coannihilation processes are included by summing up the cross sections of all the relevant modes with appropriate weights:

$$
\left\langle\sigma_{\mathrm{eff}} v\right\rangle=\sum_{i, j} r_{i} r_{j}\left\langle\sigma_{i j} v\right\rangle
$$

${ }^{\# 1}$ We use the fact that $g_{* s}(T)$ is numerically very close to $g_{*}(T)$, and approximate $g_{* s}(T) \simeq g_{*}(T)$ in our calculation, where $g_{* s}(T)$ is the effective number of massless degrees of freedom for the calculation of entropy density, which is related to the entropy density as

$$
s(T)=\frac{2 \pi^{2}}{45} g_{* s}(T) T^{3} .
$$

\footnotetext{
\#2 Because we are interested in the case where charged and neutral Winos are quite degenerate, we denote the Wino masses as $m_{\tilde{W}}$ as far as we discuss the quantities which are insensitive to the mass difference.
} 
where $i, j=\tilde{W}^{0}, \tilde{W}^{+}$and $\tilde{W}^{-}$, and

$$
r_{i}=\frac{n_{i}}{n_{\tilde{W}}}
$$

with $n_{i}$ being the number density of $i$. We assume that Winos are in kinetic equilibrium, \#3 when $T \ll m_{\tilde{W}}$,

$$
r_{\tilde{W}^{0}}=\frac{1}{1+2 e^{-\Delta m_{\tilde{W}} / T}}, \quad r_{\tilde{W}^{+}}=r_{\tilde{W}^{-}}=\frac{e^{-\Delta m_{\tilde{W}} / T}}{1+2 e^{-\Delta m_{\tilde{W}} / T}} .
$$

If the mass difference between charged and neutral Winos $\Delta m_{\tilde{W}}$ is much larger than the background temperature $T$, only the lightest neutral Wino is relevant in the annihilation process. On the other hand, the coannihilation becomes effective for the temperature $T \gtrsim$ $\Delta m_{\tilde{W}} \cdot$

For each annihilation process, thermally averaged cross section is obtained by

$$
\left\langle\sigma_{i j} v\right\rangle=\left(\frac{m_{\tilde{W}}}{4 \pi T}\right)^{3 / 2} \int d^{3} v\left(\sigma_{i j} v\right) e^{-m_{\tilde{W}} v^{2} / 4 T} .
$$

Once the temperature of the universe decreases and the neutral and charged Winos become non-relativistic, the wave functions of annihilating Wino pairs are significantly deformed by the electroweak potential generated by the electroweak gauge boson exchanges. The resultant annihilation cross sections, $\sigma_{i j} v$, are significantly enhanced or suppressed due to the Sommerfeld effect [28, 29, 30]. The Sommerfeld effect is more important for larger Wino mass, $m_{\tilde{W}} \gtrsim 1 \mathrm{TeV}$, because the electroweak potential behaves as a long range force in such a mass region. As a result, the thermally-averaged cross sections show non-trivial dependence on the cosmic temperature $T$ and the Wino mass $m_{\tilde{W}}$. A two-body system of Winos can be classified by the quantum numbers $Q$ (electric charge) and $S$ (spin), and the Sommerfeld enhancement factors are evaluated for fixed values of these quantum numbers. For each set of $(Q, S)$, possible decay modes and the decay widths are summarized in Appendix. (See Table 1.)

In Fig. 2, we show the effective annihilation cross section for several cosmic temperatures, $T=10^{-5} m_{\tilde{W}}, 10^{-4} m_{\tilde{W}}, 10^{-3} m_{\tilde{W}}$, and $T \gg m_{\tilde{W}}$. (Here and hereafter, we take $\Delta m_{\tilde{W}}=160 \mathrm{MeV}$ in our numerical calculation; even if we vary $\Delta m_{\tilde{W}}$ by $5 \mathrm{MeV}$ or so, the resultant value of the Wino abundance is almost the same. ) For comparison, we also show

\#3 The energetic Winos produced by the $X$ decay show non-trivial velocity distribution at first. However, charged Winos soon lose their energies through the electromagnetic interactions with the thermal background (in particular, electron and positron) as well as through the decay. In addition, sizable fractions of neutral Winos can be thermalized through the inelastic interactions [26, 27. In particular, as we see below, $T_{X}$ is required to be higher than $\sim \Delta m_{\tilde{W}}$ in order to realize $\Omega_{\tilde{W}}=\Omega_{\mathrm{c}}$ in the parameter region where the pair annihilation of the Wino becomes effective; in such a case, the neutral and charged Winos are efficiently converted to each other by the charged current processes in the thermal bath, and the charged Wino efficently loses its energy by the decay process (as well as the scattering processes with charged particles in the thermal bath). Thus, we expect that the Winos reach the kinetic equilibrium. 


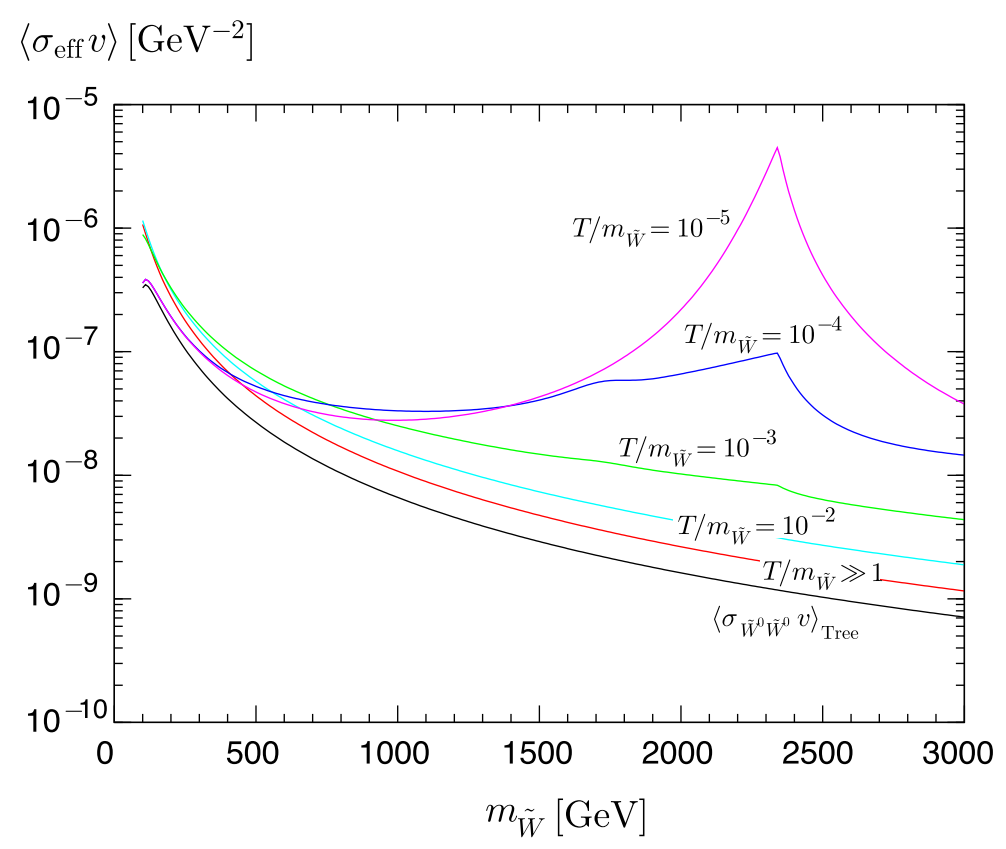

Figure 1: Thermally averaged total cross section $\left\langle\sigma_{\text {eff }} v\right\rangle$ for $T=10^{-5} m_{\tilde{W}}$ (pink line), $10^{-4} m_{\tilde{W}}$ (blue line), $10^{-3} m_{\tilde{W}}$ (green line), $10^{-2} m_{\tilde{W}}$ (light blue line), and $T \gg m_{\tilde{W}}$ (red line). For comparison, we also show the annihilation cross section of neutral Wino without the Sommerfeld effect (black line). The vertical axis is the the Wino mass, and we take $\Delta m_{\tilde{W}}=160 \mathrm{MeV}$.

the annihilation cross section of neutral Wino without the Sommerfeld effect in the same figure. Obviously, the cross section is significantly enhanced in particular for the Wino mass larger than $\sim 1 \mathrm{TeV}$, and the enhancement factor becomes larger for lower temperature. In addition, we can see the resonance structure at around $m_{\tilde{W}} \simeq 2.4 \mathrm{TeV}$, which occurs due to the existence of zero-energy bound states. The precise position of the resonance is determined by the structure of the electroweak potential, and depends on the mass difference between charged and neutral Winos, $\Delta m_{\tilde{W}}$. For larger $\Delta m_{\tilde{W}}$, the resonance peak is shifted to the heavier Wino mass.

As is seen from the Fig. 2, the annihilation cross section at the decay temperature can be significantly enhanced by the Sommerfeld effect. We stress here again that once coannihilation and Sommerfeld effects are included, the cross sections show non-trivial dependence on $T$. In order to precisely take into account these effects, we solve the Boltzmann equations numerically.

The relic density of the neutral Wino can be calculated by solving Eqs. (2.1) - (2.3) with relevant initial condition. The initial values of $n_{\tilde{W}}, n_{X}$, and $\rho_{\text {rad }}$ depend on cosmological scenarios and the properties of $X$. In the next section, we consider several well-motivated scenarios and calculate the relic density by numerically solving the Boltzmann equations. 


\section{Relic Abundance of Neutral Wino}

\subsection{Case with $X$ domination in the early universe}

First, we consider the case where the particle $X$ once dominates the universe. In such a case, at the cosmic time $t \ll \Gamma_{X}^{-1}$, the energy density of $X$ is much larger than that of radiation, while the energy density of radiation (i.e., so-called "dilute plasma") scales as $a^{-3 / 8}$ (with $a$ being the scale factor). With such an initial condition, we solve the Boltzmann equations to calculate the relic abundance of $\tilde{W}^{0}$. In the parameter region of our analysis the maximal temperature of the dilute plasma is much higher than the Wino mass; then, the pair annihilation rate is initially much larger than the expansion rate of the universe. In such a case, the production and annihilation terms in the right-hand side of Eq. (2.1) (almost) balance, so we take the initial value of the number density of the Wino as $n_{\tilde{W}}^{\text {(init) }}=$ $\sqrt{n_{\tilde{W}, \text { eq }}^{2}+N_{\tilde{W}} \Gamma_{X} n_{X}\left\langle\sigma_{\text {eff }} v\right\rangle^{-1}}$. (However, the resultant relic abundance is insensitive to the initial value of $n_{\tilde{W}}$ as far as the initial condition is set at the cosmic time with the background temperature much higher than $m_{\tilde{W}}$.) With the initial condition given above, we calculate the number density of the Wino after the completion of the decay of $X$, and evaluate the yield variable of the Wino

$$
Y_{\tilde{W}} \equiv \frac{n_{\tilde{W}}}{s},
$$

where $s$ is the entropy density. Using the fact that $Y_{\tilde{W}}$ becomes constant of time at low enough temperature, we calculate the density parameter as

$$
\Omega_{\tilde{W}}=m_{\tilde{W}}\left[Y_{\tilde{W}}\right]_{t \gg \Gamma_{X}^{-1}}\left(\frac{\rho_{\text {crit }}}{s_{\text {now }}}\right)^{-1},
$$

where $\rho_{\text {crit }}$ is the critical density of the universe while $s_{\text {now }}$ is the present entropy density, and their ratio is given by $\rho_{\text {crit }} / s_{\text {now }} \simeq 3.6 h^{2} \times 10^{-9} \mathrm{GeV}$, with $h$ being the Hubble constant in units of $100 \mathrm{~km} / \mathrm{sec} / \mathrm{Mpc}$. (In our numerical calculation, we use $h=0.697$ [31].) Comparing $\Omega_{\tilde{W}}$ with the present density parameter of dark matter, we derive constraints on the model parameters. We use the following value of the dark matter density as the canonical value [31]:

$$
\Omega_{\mathrm{c}} h^{2}=0.1146
$$

In the case where $X$ once dominates the universe, the relic density of the Wino depends on the following parameters: $\Gamma_{X}$ (decay rate of $X$ ), $m_{X}$ (mass of $X$ ), $m_{\tilde{W}}$ (Wino mass), and $N_{\tilde{W}}$ (averaged number of the Winos produced by the decay of one $X$ ). In particular, the relic density depends on the decay rate $\Gamma_{X}$. To discuss the dependence on $\Gamma_{X}$, it is convenient to define the "decay temperature" as

$$
T_{X} \equiv\left(\frac{10}{g_{*}\left(T_{X}\right) \pi^{2}} M_{\mathrm{Pl}}^{2} \Gamma_{X}^{2}\right)^{1 / 4}
$$




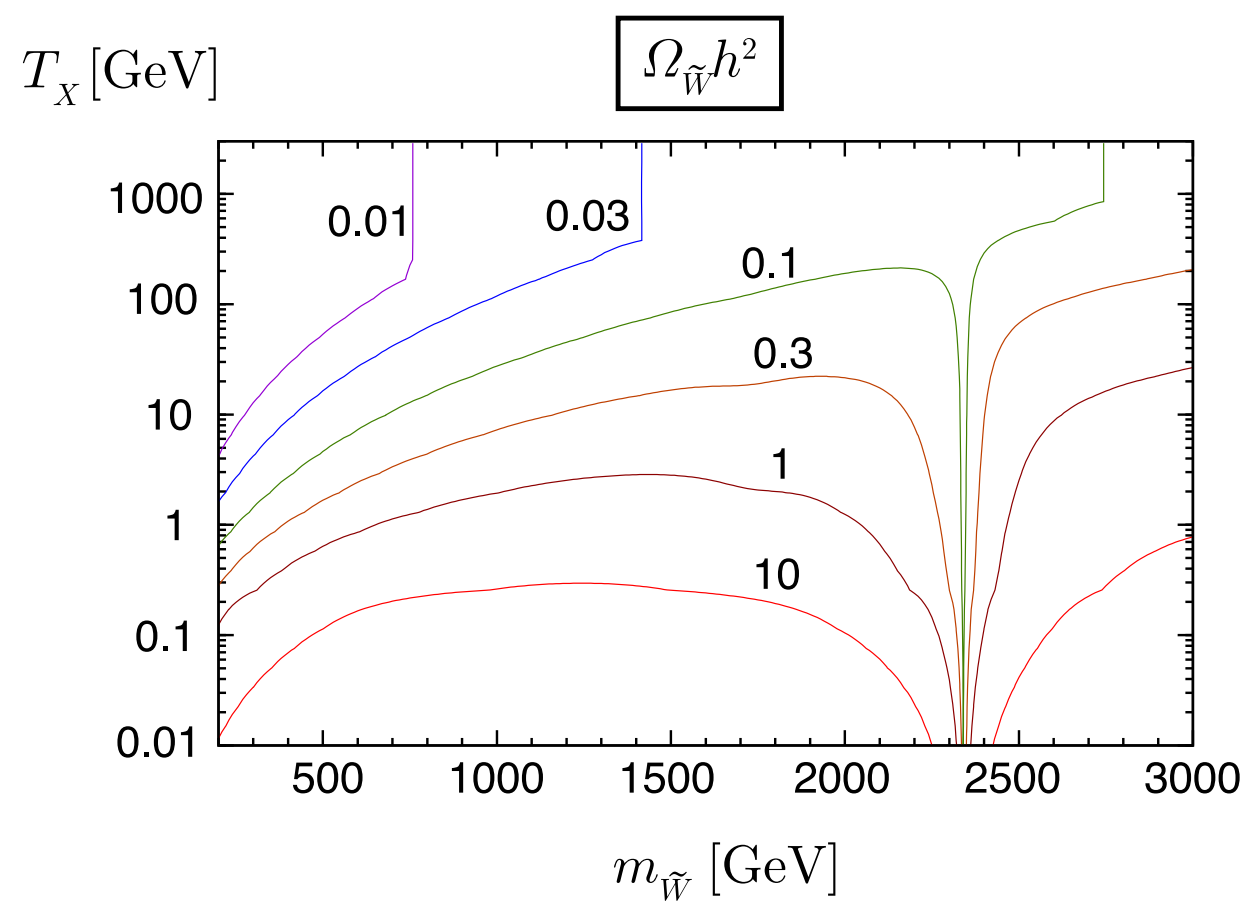

Figure 2: Contours of constant $\Omega_{\tilde{W}} h^{2}$ for $N_{\tilde{W}}=1$ and $m_{X}=5 m_{\tilde{W}}$ for the case where $X$ once dominates the universe. Numbers in the figure are $\Omega_{\tilde{W}} h^{2}$.

where $M_{\mathrm{Pl}} \simeq 2.4 \times 10^{18} \mathrm{GeV}$ is the reduced Planck scale. Notice that $T_{X}$ corresponds to the cosmic temperature at the time of $X$ decay.

In Fig. 2, we show the contours of constant $\Omega_{\tilde{W}} h^{2}$ for the case with $N_{\tilde{W}}=1$. (In this subsection, we take $m_{X} / m_{\tilde{W}}=5$; even if we vary this ratio, the resultant value of $\Omega_{\tilde{W}} h^{2}$ is almost unchanged if the annihilation of the Winos becomes effective.) As one can see, $\Omega_{\tilde{W}} h^{2}$ is insensitive to $T_{X}$ when the decay temperature is high, while $\Omega_{\tilde{W}} h^{2}$ increases as $T_{X}$ becomes lower when the decay temperature is relatively low. Such behaviors can be easily understood. With high enough decay temperature, the Winos are still in chemical equilibrium after the completion of the $X$ decay, and hence the relic abundance of the Wino is given by the thermal relic density. On the contrary, with low decay temperature, the Winos cannot be in chemical equilibrium. Even in such a case, the pair annihilation of the Wino proceeds as far as the annihilation rate is larger than the expansion rate of the universe. In such a case, the yield value of the Wino is approximated by

$$
Y_{\tilde{W}} \sim \min \left[\frac{3 \Gamma_{X}}{\left\langle\sigma_{\mathrm{eff}} v\right\rangle s\left(T_{X}\right)}, \frac{N_{\tilde{W}} n_{X}\left(T_{X}\right)}{s\left(T_{X}\right)}\right],
$$

where $n_{X}\left(T_{X}\right)$ represents the number density of $X$ just before the decay. Notice that the first (second) term in the right-hand side of Eq. (3.14) is relevant for the case where the effect of the annihilation of the Wino is effective (ineffective). The first term, which is approximately proportional to $T_{X}^{-1}$, is smaller than the second one, resulting in the enhancement of $\Omega_{\tilde{W}} h^{2}$ 


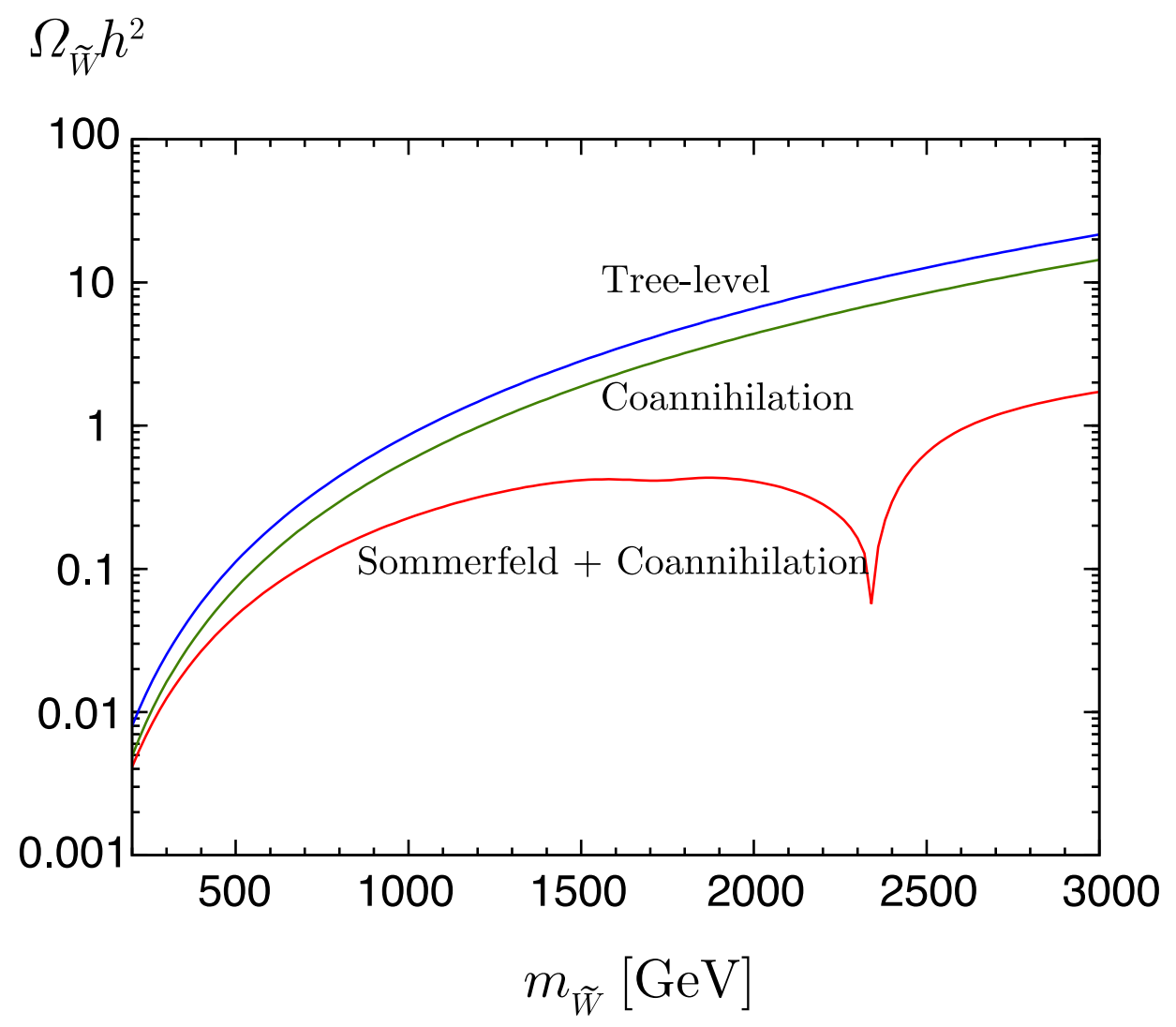

Figure 3: $\Omega_{\tilde{W}} h^{2}$ as a function of $m_{\tilde{W}}$ for $N_{\tilde{W}}=1$ and $T_{X}=10 \mathrm{GeV}$ with Sommerfeld and coannihilation effects (red line), with coannihilation effect only (green line) and without neither Sommerfeld nor coannihilation effects (blue line).

with lower decay temperature.

In order to realize the Wino dark matter scenario (i.e., $\Omega_{\tilde{W}}=\Omega_{\mathrm{c}}$ ), relatively high value of $T_{X}$ is needed. For $m_{\tilde{W}}=300 \mathrm{GeV}(500 \mathrm{GeV}, 1 \mathrm{TeV}, 2 \mathrm{TeV}), T_{X}=1.3 \mathrm{GeV}(4.0 \mathrm{GeV}$, $23 \mathrm{GeV}, 150 \mathrm{GeV}$ ) is necessary. If $T_{X}$ is lower, the universe is overclosed. This has an important implication when the cosmological moduli fields play the role of $X$, as we discuss below. In addition, we can see a significant suppression of $\Omega_{\tilde{W}} h^{2}$ when $m_{\tilde{W}} \simeq 2.4 \mathrm{TeV}$. Such a suppression is due to the significant enhancement of the annihilation cross section by the Sommerfeld effect.

In order to see the importance of Sommerfeld enhancement, we also calculate $\Omega_{\tilde{W}} h^{2}$ neglecting the Sommerfeld enhancement. In addition, for comparison, we also show the result without the effect of coannihilation, for which we use $\left\langle\sigma_{\text {eff }} v\right\rangle=\left\langle\sigma_{\tilde{W}^{0} \tilde{W}^{0} \rightarrow W^{+} W^{-}} v\right\rangle$ (without Sommerfeld effect). The results are shown in Fig. 3 for $N_{\tilde{W}}=1$ and $T_{X}=10 \mathrm{GeV}$. The Sommerfeld effect significantly changes $\Omega_{\tilde{W}} h^{2}$ when $m_{\tilde{W}} \gtrsim 1 \mathrm{TeV}$.

In the case where $X$ dominates the universe, the $N_{\tilde{W}}$ dependence of $\Omega_{\tilde{W}} h^{2}$ is very weak unless $N_{\tilde{W}}$ is significantly suppressed. In Fig. 过, we plot contours of constant $\Omega_{\tilde{W}} h^{2}$ with 


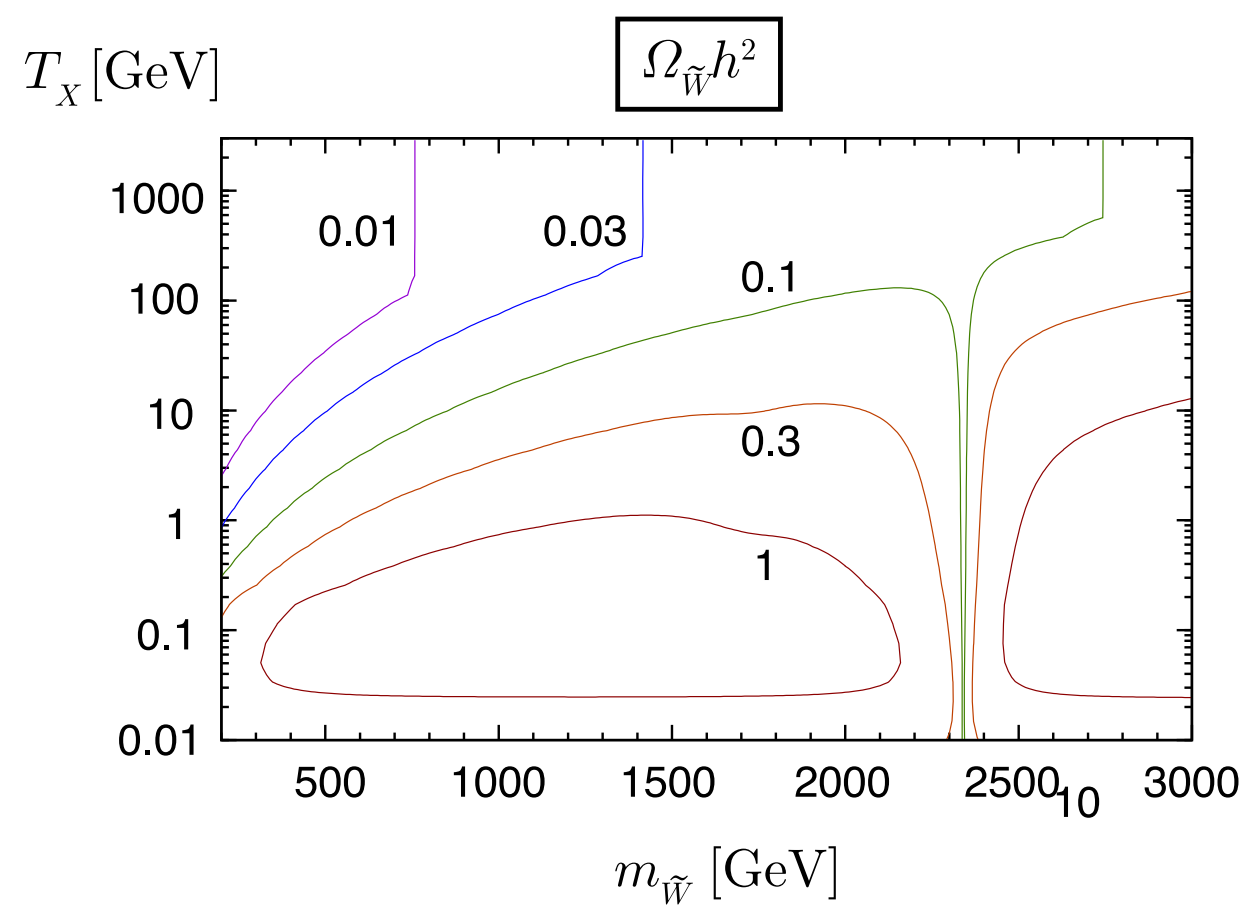

Figure 4: Same as Fig. 2, except for $N_{\tilde{W}}=10^{-6}$.

$N_{\tilde{W}}=10^{-6}$. Even with such a small value of $N_{\tilde{W}}$, the contour of $\Omega_{\tilde{W}}=\Omega_{c}$ does not change much compared to that given in Fig. 2. Thus, unless $N_{\tilde{W}} \ll 10^{-6}, T_{X}$ should be higher than $0.6 \mathrm{GeV}(2 \mathrm{GeV}, 10 \mathrm{GeV}$, and $90 \mathrm{GeV})$ for $m_{\tilde{W}}=300 \mathrm{GeV}\left(m_{\tilde{W}}=500 \mathrm{GeV}, m_{\tilde{W}}=1 \mathrm{TeV}\right.$, and $m_{\tilde{W}}=2 \mathrm{TeV}$ ). In Fig. [5, we show the contours of the upper bound on $N_{\tilde{W}}$ on the $m_{\tilde{W}}$ vs. $T_{X}$ plane, requiring $\Omega_{\tilde{W}}<\Omega_{\mathrm{c}}$. As a result, we can see that $N_{\tilde{W}} \lesssim O\left(10^{-8}\right)$ is required if $T_{X}$ is so small that the pair annihilation of the Winos is ineffective.

One of the important applications of our study is to the case with weakly interacting late decaying scalar fields, like scalar fields responsible for the SUSY breaking (i.e., socalled the Polonyi field) and the moduli fields in string theory. (Hereafter, we call them "moduli fields.") Moduli fields are expected to have interactions suppressed by the Planck scale. A modulus field may have a large initial amplitude. Because of the weakness of the interaction, the lifetime of the modulus may be so long that its decay occurs at a late stage of the evolution of the universe. It is well known that the late-time decay of the modulus field is dangerous because it destroys the light elements produced by the BBN reactions, resulting in the spoil of the success of the BBN scenario. Such a problem can be avoided if the decay rate of the modulus is somehow enhanced so that the lifetime of the modulus becomes shorter than $\sim 1 \mathrm{sec}$. Even so, the decay of the modulus field produces significant amount of superparticles, which may result in the overproduction of the LSP. With the Wino LSP, this problem may be avoided.

The decay rate of a modulus field depends on how it interacts with the MSSM fields. For 


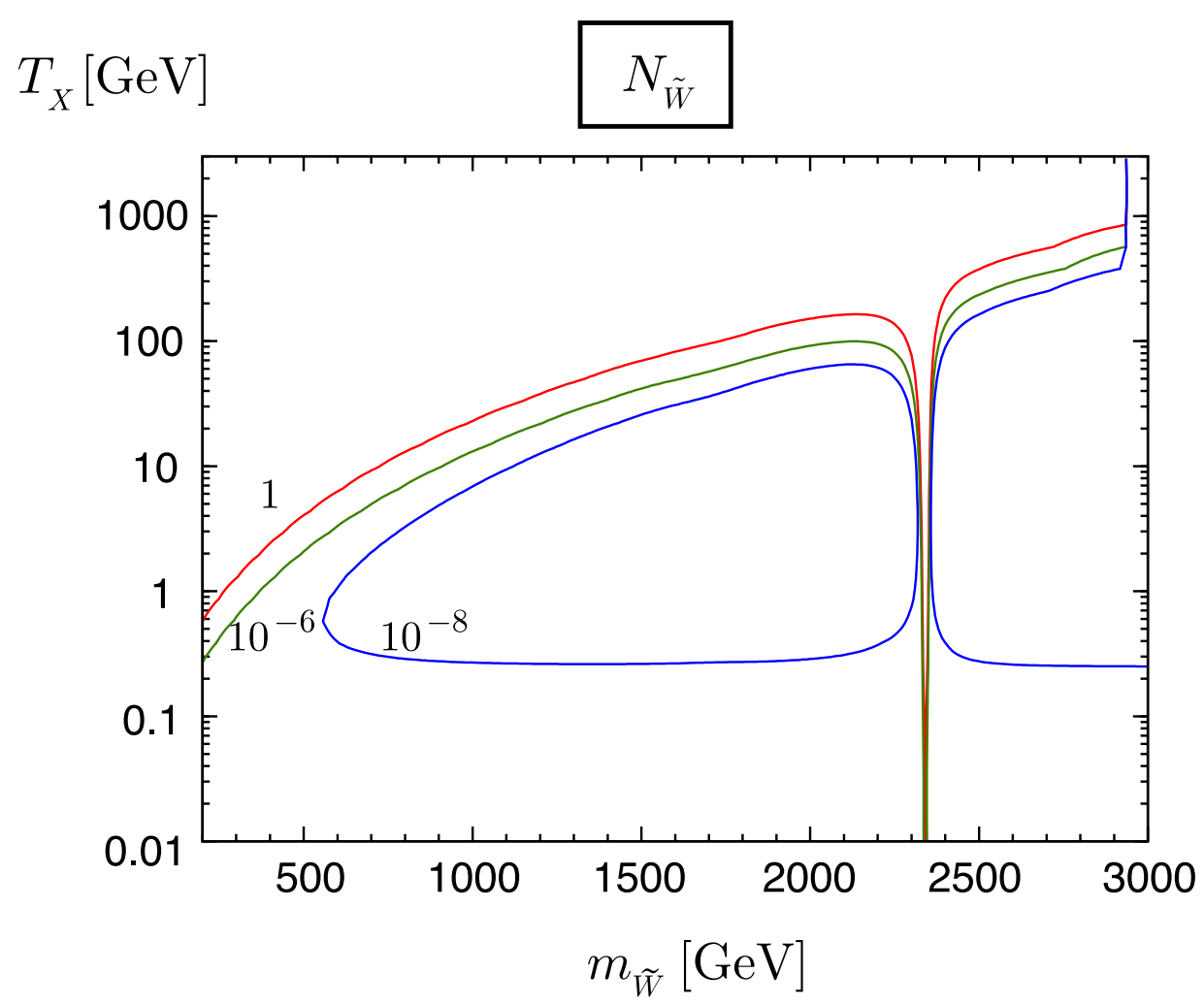

Figure 5: Contours of $\Omega_{\tilde{W}}=\Omega_{c}$ for $N_{\tilde{W}}=1$ (blue line), $N_{\tilde{W}}=10^{-6}$ (green line) and $N_{\tilde{W}}=10^{-8}$ (red line).

example, it may interact as

$$
\mathcal{L}_{\text {int }}=\frac{\lambda_{\mathrm{G}}}{M_{\mathrm{Pl}}} \int d^{2} \theta \hat{X} \hat{\mathcal{W}}^{\alpha} \hat{\mathcal{W}}_{\alpha}+\text { h.c. },
$$

where the "hat" stands for superfields, $\hat{\mathcal{W}}$ is the gauge field strength superfield, and $\lambda_{\mathrm{G}}$ is a coupling constant. Then, the scalar component $X$ decays into a pair of vector boson $V$ with the following decay rate:

$$
\Gamma_{X \rightarrow V V}=\frac{N_{\mathrm{f}} \lambda_{\mathrm{G}}^{2}}{4 \pi} \frac{m_{X}^{3}}{M_{\mathrm{Pl}}^{2}},
$$

where $N_{\mathrm{f}}$ is the number of the possible final states; for example, $N_{\mathrm{f}}=N^{2}-1$ for an $\mathrm{SU}(N)$ gauge group. (Here, we assume that the vector boson is much lighter than $X$.) In addition, with the interaction given in Eq. (3.15), $X$ may decay into a pair of gauginos $\lambda$. The decay rate is model-dependent, and is typically $\Gamma_{X \rightarrow \lambda \lambda} \sim \Gamma_{X \rightarrow V V}$ [33, 34]. Approximating $\Gamma_{X} \sim \Gamma_{X \rightarrow V V}$, the decay temperature is estimated to be

$$
T_{X} \sim 0.01 \mathrm{GeV} \times \lambda_{\mathrm{G}}\left(\frac{m_{X}}{100 \mathrm{TeV}}\right)^{3 / 2},
$$


where we have used $N_{\mathrm{f}}=12$, which is the number of the gauge bosons in the standard model. For $m_{\tilde{W}}=300 \mathrm{GeV}(1 \mathrm{TeV})$, the $\lambda_{\mathrm{G}}$-parameter is required to be larger than 430,130 , and $42(7600,2300$, and 74$)$ for $m_{X}=10 \mathrm{TeV}, 100 \mathrm{TeV}$, and $1000 \mathrm{TeV}$, respectively, where we have assumed $N_{\tilde{W}} \sim 1$. It seems that if such a modulus with $\lambda_{G} \sim O(1)$ once dominates the universe it tends to cause overproduction of the Winos. In order for the Winos not to overclose the universe, large $m_{X}$ or enhanced $\lambda_{\mathrm{G}}$ is needed.

\subsection{Case with gravitino}

Next, we consider the case where gravitino plays the role of $X$. Even though the gravitino may not dominate the universe, a significant amount of $\tilde{W}$ may be produced by its decay.

The gravitino is the superpartner of graviton, and it couples to the supercurrent so it interacts with all the supermultiplets. If unstable, the gravitino decays into an ordinary (visible-sector) particle and its superpartner. Thus, decay of the gravitino results in the production of the LSP because all the produced superparticles cascade down to the LSP. The interactions of the gravitino are suppressed by inverse powers of the Planck scale so that the decay rate of the gravitino is extremely small. It is well known that the late-time decay of the gravitino may spoil the success of the BBN if the lifetimes of the gravitino is longer than $\sim 1 \sec [6,7,32$. In order to avoid such a problem, we concentrate on the case where the lifetime is shorter than $\sim 1 \mathrm{sec}$, which is realized if the gravitino mass is larger than $\sim O(10 \mathrm{TeV})$. Assuming that the gaugino masses are at the $\mathrm{TeV}$ scale and are much smaller than the gravitino mass, the lifetime of the gravitino is estimated as

$$
\tau_{3 / 2}=0.4 \mathrm{sec} \times N_{\mathrm{G}}^{-1}\left(\frac{m_{3 / 2}}{100 \mathrm{TeV}}\right)^{-3},
$$

where $m_{3 / 2}$ is gravitino mass. In addition, $N_{\mathrm{G}}$ is the number of gauge multiplets to which the gravitino decays. (In the following numerical study, we take the MSSM value of $N_{\mathrm{G}}=12$.) We also assume that the superparticles other than gauginos are as heavy as the gravitino so that the decay modes into those superparticles are negligible.

Even though the gravitino is very weakly interacting, gravitinos are produced by scattering processes of particles in thermal bath. The abundance of the gravitino (before its decay) is approximately proportional to the reheating temperature after inflation; for the case where the gravitino mass is significantly larger than gaugino masses, the yield variable of the gravitino, which is defined as $Y_{3 / 2}=n_{3 / 2} / s$ with $n_{3 / 2}$ being the number density of the gravitino, is given by $[32] \# 4$

$$
\left[Y_{3 / 2}\right]_{t \ll \tau_{3 / 2}} \simeq 2.3 \times 10^{-14} \times T_{\mathrm{R}}^{(8)}\left[1+0.015 \ln T_{\mathrm{R}}^{(8)}-0.0009 \ln ^{2} T_{\mathrm{R}}^{(8)}\right],
$$

\footnotetext{
\#4 If there exists condensation of a scalar field (like inflaton, moduli fields, and so on), the gravitino may be produced by the decay process $33,34,35$. We assume that the abundance of the gravitino from such a decay process, which is highly model-dependent, is negligible.
} 


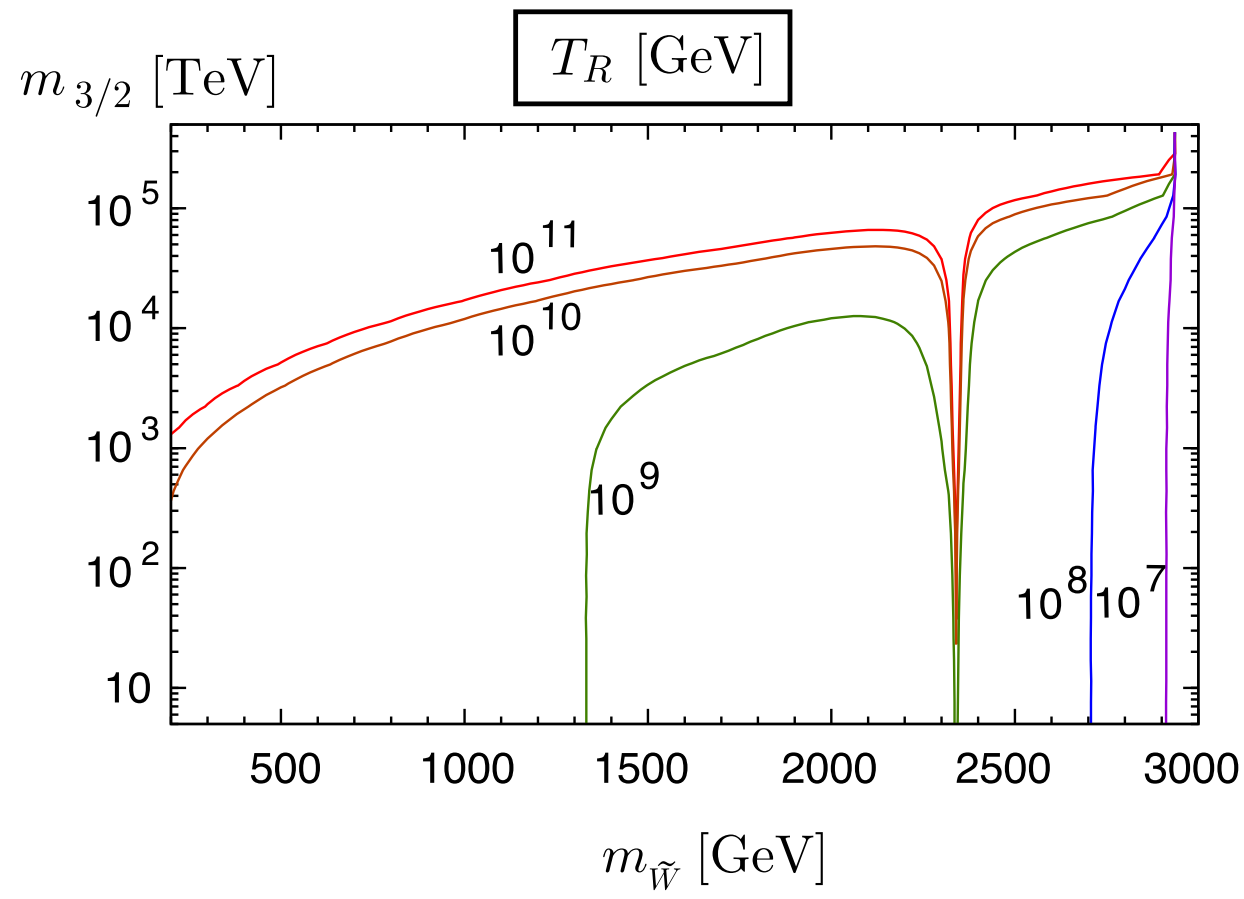

Figure 6: Contours of $\Omega_{\tilde{W}}=\Omega_{c}$ for several values of the reheating temperature. Here we take $N_{\mathrm{G}}=12$. Numbers in the figure are $T_{\mathrm{R}}$ in units of $\mathrm{GeV}$.

where $T_{\mathrm{R}}^{(8)} \equiv T_{\mathrm{R}} / 10^{8} \mathrm{GeV}$, with $T_{\mathrm{R}}$ being the reheating temperature after inflation, \#5 which is related to the decay rate of the inflaton $\Gamma_{\text {inf }}$ as

$$
T_{\mathrm{R}} \equiv\left(\frac{10}{g_{*}\left(T_{\mathrm{R}}\right) \pi^{2}} M_{\mathrm{Pl}}^{2} \Gamma_{\mathrm{inf}}^{2}\right)^{1 / 4} .
$$

We calculate the density parameter of the Wino by numerically solving the Boltzmann equations given in Section 2. Because the primordial abundance of the gravitino is approximately proportional to the reheating temperature, $\Omega_{\tilde{W}}$ also increases with higher reheating temperature.

In Fig. 6, we show the contours of $\Omega_{\tilde{W}}=\Omega_{c}$ for several values of $T_{\mathrm{R}}$ on $m_{\tilde{W}}$ vs. $m_{3 / 2}$ plane \#6 The contour of $\Omega_{\tilde{W}}=\Omega_{\mathrm{c}}$ is almost independent of the reheating temperature if $T_{\mathrm{R}}$ is high enough. This is due to the fact that, with high enough reheating temperature and large enough gravitino mass, the Wino abundance at the time of the gravitino decay is so large that the pair annihilation of Wino becomes effective. Then, as discussed in the previous subsection, $\Omega_{\tilde{W}}$ is determined by the decay temperature which is determined by $m_{3 / 2}$ and becomes insensitive to the primordial abundance of the gravitino. On the contrary, if the

\footnotetext{
${ }^{\# 5}$ Here, we assume that there is no significant entropy production after inflation.

${ }^{\# 6}$ In the anomaly-mediation scenario with $m_{\tilde{W}} \sim O(100 \mathrm{GeV}-1 \mathrm{TeV})$, the gravitino mass is expected to be of $O(100 \mathrm{TeV})$. Here, however, we vary $m_{3 / 2}$ up to $O\left(10^{5} \mathrm{TeV}\right)$ to provide information about the case with extremely heavy gravitino.
} 


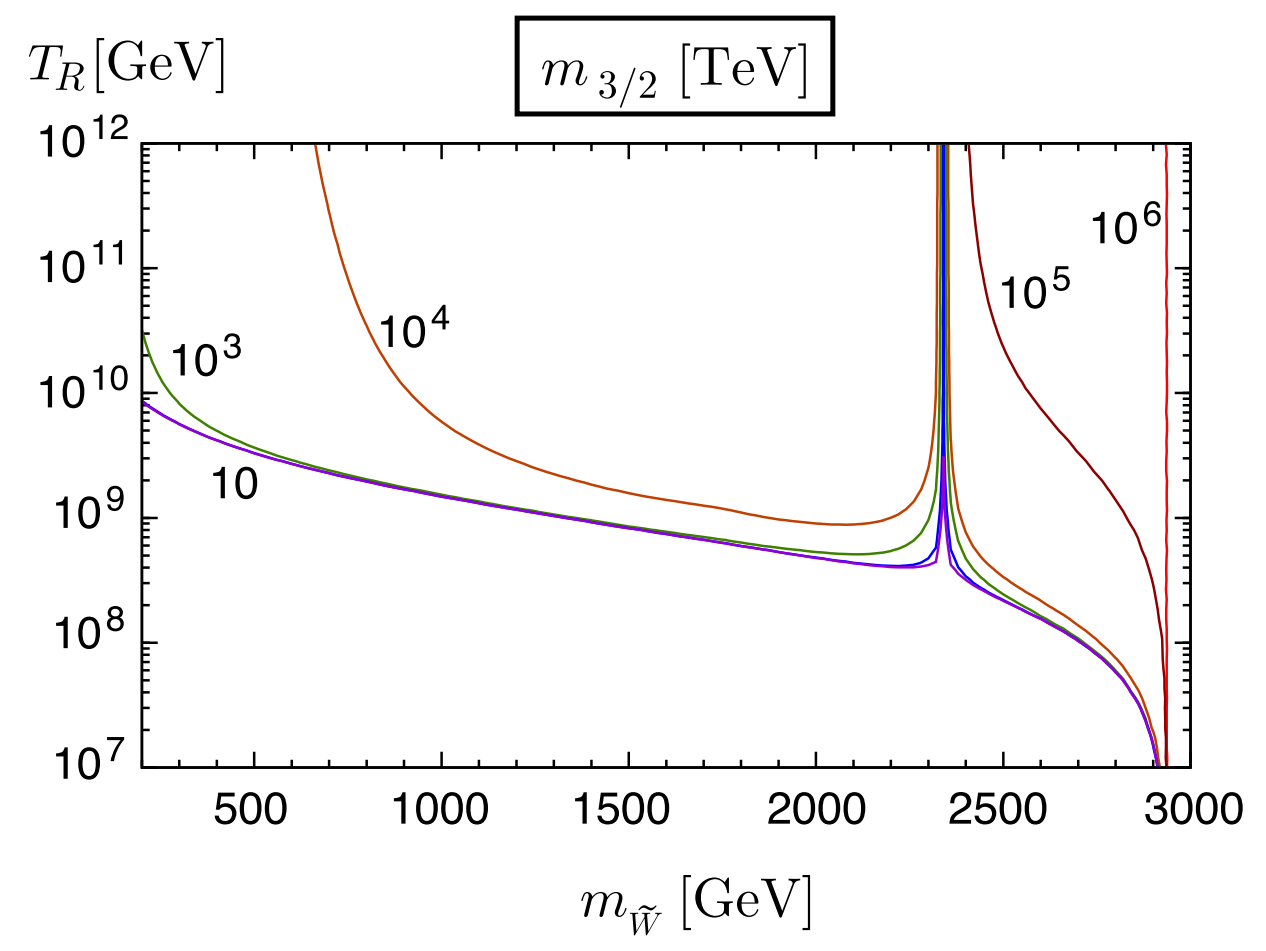

Figure 7: Upper bound on the reheating temperature as a function of the Wino mass for several values of the gravitino mass. Numbers in the figure are the gravitino mass in units of $\mathrm{TeV}$. (The bound for $m_{3 / 2}=10^{2} \mathrm{TeV}$ (blue line) almost overlaps with that of $m_{3 / 2}=10 \mathrm{TeV}$.)

gravitino mass is smaller than $\sim 10^{3} \mathrm{TeV}$, the contour of $\Omega_{\tilde{W}}=\Omega_{\mathrm{c}}$ becomes insensitive to the gravitino mass. This is because, if the gravitino mass is small, the decay temperature of the gravitino becomes so low that the annihilation of the Wino is inefficient. Then, almost all Winos produced by the gravitino decay survive until today, and the relic density of the Wino is approximately given by the sum of thermal relic density and the non-thermal one from the gravitino decay: $Y_{\tilde{W}} \simeq Y_{\tilde{W}}^{(\text {th) }}+\left[Y_{3 / 2}\right]_{t \ll \tau_{3 / 2}}$, with $Y_{\tilde{W}}^{\text {(th) }}$ being the thermal abundance of the Wino. The thermal relic density becomes larger than the present dark matter density if $m_{\tilde{W}} \gtrsim 2.9 \mathrm{TeV}$, so the Wino mass larger than $\sim 2.9 \mathrm{TeV}$ is forbidden irrespective of $T_{\mathrm{R}}$. On the contrary, with smaller Wino mass, we obtain upper bound on the reheating temperature by requiring $\Omega_{\tilde{W}}<\Omega_{c}$, using the fact that the primordial number density of the gravitino is approximately proportional to $T_{\mathrm{R}}$. Such a bound is shown in Fig. 7 for several values of the gravitino mass.

One implication of our result is on the leptogenesis scenario [36]. The leptogenesis scenario requires the reheating temperature to be higher than $\sim 10^{9} \mathrm{GeV}$ in order to generate large enough amount of the baryon asymmetry of the universe [37, 38]. We have seen that such a high reheating temperature can be realized in the Wino LSP case. 


\subsection{Case with SUSY Peccei-Quinn model}

The Peccei-Quinn (PQ) mechanism [39, 40] is an attractive solution to the strong CP problem. If we embed the PQ mechanism into supersymmetric model, there exists the fermionic superpartner of the axion called axino. Axinos are copiously produced in the thermal bath of early universe and decay into MSSM particles at a late stage of the cosmic expansion. In this subsection, we consider the case where the axino $\tilde{a}$ plays the role of $X$, which is embedded in the axion multiplet $\hat{\mathcal{A}}$ a:\#7

$$
\hat{\mathcal{A}}=\frac{1}{\sqrt{2}}(\sigma+i a)+\sqrt{2} \theta \tilde{a}+(F \text {-term }) .
$$

(In this subsection, the field $X$ is denoted as $\tilde{a}$.)

To make our discussion concrete, we assume that the PQ charges of all the MSSM fields are zero, and that the PQ fermions are embedded into full multiplets of $S U(5)$ grand unified gauge group. In such a model, denoting the PQ scale as $f_{a}$, the axion multiplet $\hat{\mathcal{A}}$ has the following interaction term:

$$
\mathcal{L}_{\text {int }}=\frac{1}{4 \sqrt{2} \pi f_{a}} \int d^{2} \theta\left[\alpha_{3} \hat{\mathcal{A}} \hat{\mathcal{G}}^{\alpha} \hat{\mathcal{G}}_{\alpha}+\alpha_{2} \hat{\mathcal{A}} \hat{\mathcal{W}}^{\alpha} \hat{\mathcal{W}}_{\alpha}+\frac{5}{3} \alpha_{1} \hat{\mathcal{A}} \hat{\mathcal{B}}^{\alpha} \hat{\mathcal{B}}_{\alpha}\right]+\text { h.c. }
$$

where $\hat{\mathcal{G}}, \hat{\mathcal{W}}$, and $\hat{\mathcal{B}}$ are field strength superfields for $S U(3)_{C}, S U(2)_{L}$, and $U(1)_{Y}$, respectively, and $\alpha_{a} \equiv g_{a}^{2} / 4 \pi$ with $g_{a}(a=1-3)$ being gauge coupling constants. (The summation over the gauge indices are implicit for $S U(3)_{C}$ and $S U(2)_{L}$.)

As in the case of the gravitino, the primordial abundance of the axino depends on the reheating temperature after inflation [46]. We adopt the yield variable of axino (i.e., $Y_{\tilde{a}}=$ $n_{\tilde{a}} / s$, with $n_{\tilde{a}}$ being the number density of axino) evaluated in [47]:

$$
\left[Y_{\tilde{a}}\right]_{t \ll \Gamma_{\tilde{a}}^{-1}} \simeq \min \left[Y_{\tilde{a}}^{(\mathrm{eq})}, 0.20 \times \alpha_{3}^{3} \ln \left(\frac{0.0977}{\alpha_{3}}\right)\left(\frac{T_{\mathrm{R}}}{10^{7} \mathrm{GeV}}\right)\left(\frac{f_{a}}{10^{11} \mathrm{GeV}}\right)^{-2}\right],
$$

where $Y_{\tilde{a}}^{(\mathrm{eq})} \simeq 1.8 \times 10^{-3}$ is the thermal abundance of axino. If the axino is stable, the axinos produced in the early universe survive until today. Then, in order not to overclose the universe with the mass density of axino, severe upper bound on the reheating temperature is obtained [46, 47, 48, 49]. Even if all the LSPs produced by the axino decay remain until today, which is the case if the annihilation cross section of the LSP is small, a stringent upper bound on $T_{\mathrm{R}}$ still exists. With a large annihilation cross section of dark matter, this problem may be avoided [50].

One of the important parameters to calculate the relic Wino abundance produced by the axino decay is the decay rate of axino. In the present setup, the axino decays dominantly

${ }^{\# 7}$ The real scalar component of $\hat{\mathcal{A}}$ (i.e., saxion) may also cause cosmological difficulties. Cosmology with saxion depends on the model of PQ sector as well as on thermal history. See [41, 42, 43, 44, 45] for more detailed discussion. 
into gauge boson and gaugino pair; with the interaction terms given in Eq. (3.22), the decay rate is given by

$$
\Gamma_{\tilde{a}}=\Gamma_{\tilde{a} \rightarrow g \tilde{g}}+\Gamma_{\tilde{a} \rightarrow W^{ \pm} \tilde{W}^{\mp}}+\Gamma_{\tilde{a} \rightarrow Z \tilde{W}^{0}}+\Gamma_{\tilde{a} \rightarrow \gamma \tilde{W}^{0}}+\Gamma_{\tilde{a} \rightarrow Z \tilde{B}}+\Gamma_{\tilde{a} \rightarrow \gamma \tilde{B}},
$$

where, if kinematically allowed [17],

$$
\begin{aligned}
\Gamma_{\tilde{a} \rightarrow g \tilde{g}} & =\frac{8 \alpha_{3}^{2}}{128 \pi^{3}} \frac{m_{\tilde{a}}^{3}}{f_{a}{ }^{2}}\left(1-y_{\tilde{g}}^{2}\right)^{3}, \\
\Gamma_{\tilde{a} \rightarrow W^{ \pm} \tilde{W}^{\mp}} & =\frac{2 \alpha_{2}^{2}}{128 \pi^{3}} \frac{m_{\tilde{a}}^{3}}{f_{a}{ }^{2}} K\left(y_{\tilde{W}}, y_{W}\right), \\
\Gamma_{\tilde{a} \rightarrow Z \tilde{W}^{0}} & =\frac{\alpha_{2}^{2} \cos ^{2} \theta_{W}}{128 \pi^{3}} \frac{m_{\tilde{a}}{ }^{3}}{f_{a}^{2}} K\left(y_{\tilde{W}}, y_{Z}\right), \\
\Gamma_{\tilde{a} \rightarrow \gamma \tilde{W}^{0}} & =\frac{\alpha_{2}^{2} \sin ^{2} \theta_{W}}{128 \pi^{3}} \frac{m_{\tilde{a}}{ }^{3}}{f_{a}{ }^{2}}\left(1-y_{\tilde{W}}{ }^{2}\right)^{3}, \\
\Gamma_{\tilde{a} \rightarrow Z \tilde{B}} & =\left(\frac{5}{3}\right)^{2} \frac{\alpha_{1}^{2} \sin ^{2} \theta_{W}}{128 \pi^{3}} \frac{m_{\tilde{a}}{ }^{3}}{f_{a}{ }^{2}} K\left(y_{\tilde{B}}, y_{Z}\right), \\
\Gamma_{\tilde{a} \rightarrow \gamma \tilde{B}} & =\left(\frac{5}{3}\right)^{2} \frac{\alpha_{1}^{2} \cos ^{2} \theta_{W}}{128 \pi^{3}} \frac{m_{\tilde{a}}{ }^{3}}{f_{a}{ }^{2}}\left(1-y_{\tilde{B}}{ }^{2}\right)^{3},
\end{aligned}
$$

where $\theta_{W}$ is the Weinberg angle, $y_{I} \equiv m_{I} / m_{\tilde{a}}$ denotes the mass of the particle $I$ normalized by the axino mass $m_{\tilde{a}}$, and

$$
K\left(y_{1}, y_{2}\right)=\sqrt{1+y_{1}^{4}+y_{2}^{4}-2 y_{1}^{2}-2 y_{2}^{2}-2 y_{1}^{2} y_{2}^{2}}\left[\left(1-y_{1}^{2}\right)^{2}+3 y_{1} y_{2}^{2}-\frac{y_{2}^{2}}{2}\left(1+y_{1}^{2}+y_{2}^{2}\right)\right]
$$

The decay temperature, and hence the resultant Wino abundance, depend on the mass spectrum of superparticles, in particular, that of gauginos. In the case where the gluino is lighter than the axino, the axino dominantly decays into gluon and gluino pair. In such a case, the decay rate of the axino becomes relatively large. In the opposite case, the dominant decay modes of the axino are $\tilde{a} \rightarrow W^{ \pm} \tilde{W}^{\mp}, \tilde{a} \rightarrow Z \tilde{W}^{0}$, and $\tilde{a} \rightarrow \gamma \tilde{W}^{0}$, and the decay rate of the axino is suppressed. So, in the latter case, $\Omega_{\tilde{W}}$ becomes larger compared to the former case.

In Fig. 8, we show the contours of constant $\Omega_{\tilde{W}} h^{2}$ for the case with high enough reheating temperature $\left(\left[Y_{\tilde{a}}\right]_{t \ll \Gamma_{\tilde{a}}^{-1}}=Y_{\tilde{a}}^{(\mathrm{eq})}\right)$, taking $m_{\tilde{W}}: m_{\tilde{B}}: m_{\tilde{g}}: m_{\tilde{a}}=1: 3: 7: 10$. (The gaugino masses are assumed to obey the anomaly-mediation relation [8, 9].) With such a mass spectrum, the axino dominantly decays into gluon and gluino pair. Then, $\Omega_{\tilde{W}}=\Omega_{c}$ is realized with $f_{a}=1.9 \times 10^{11} \mathrm{GeV}\left(1.3 \times 10^{11} \mathrm{GeV}, 6.3 \times 10^{10} \mathrm{GeV}, 2.7 \times 10^{10} \mathrm{GeV}\right)$ for $m_{\tilde{W}}=300 \mathrm{GeV}\left(m_{\tilde{W}}=500 \mathrm{GeV}, m_{\tilde{W}}=1 \mathrm{TeV}\right.$, and $\left.m_{\tilde{W}}=2 \mathrm{TeV}\right)$.

If we consider the case where the decay mode into the gluon and gluino pair is kinematically blocked, the decay rate is suppressed. In such a case, the PQ scale which realizes the Wino dark matter becomes smaller. In Fig. 9, we show the result taking $m_{\tilde{W}}: m_{\tilde{B}}: m_{\tilde{g}}$ : 


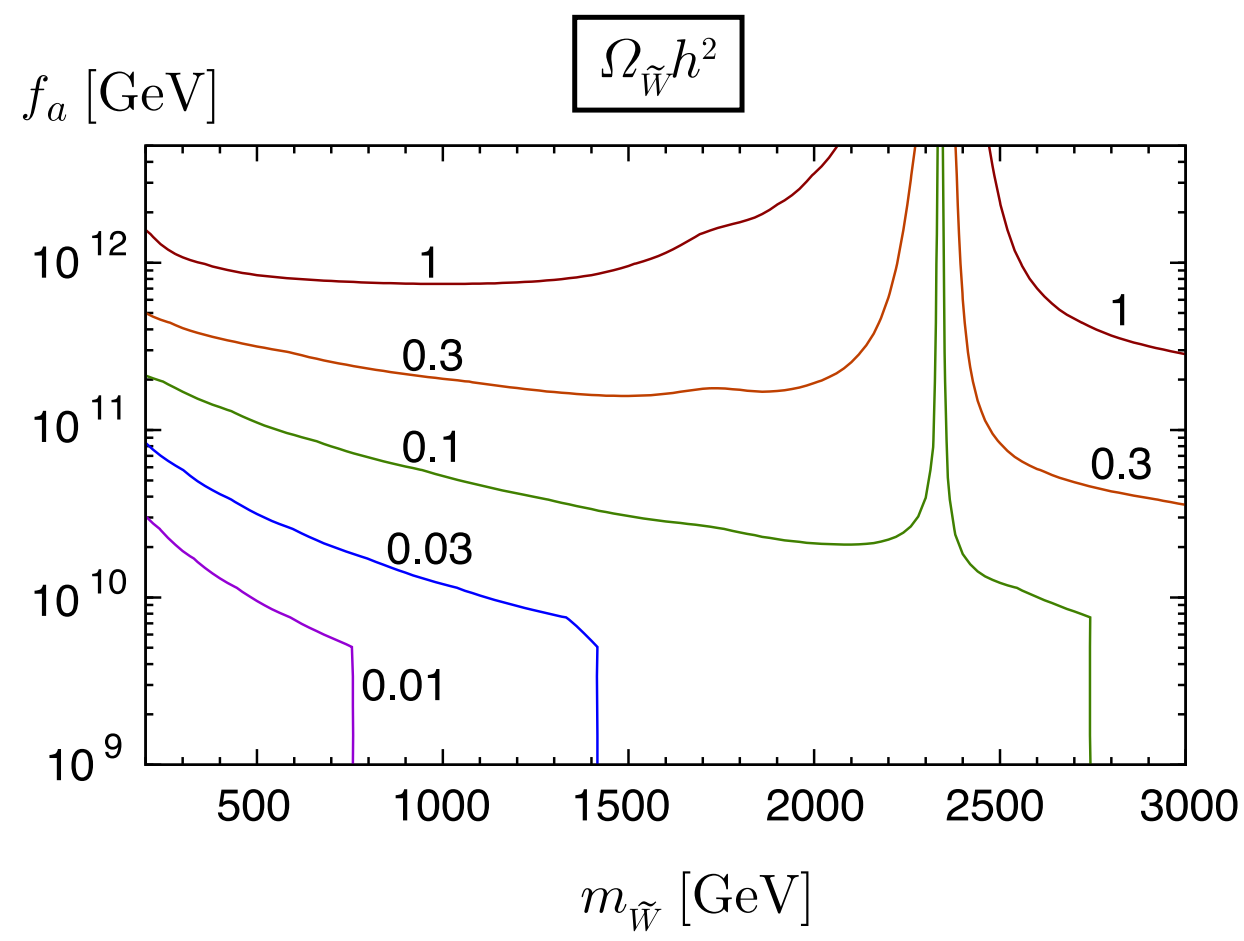

Figure 8: Contour of $\Omega_{\tilde{W}} h^{2}$ of the present universe for mass ratio $m_{\tilde{W}}: m_{\tilde{B}}: m_{\tilde{g}}: m_{\tilde{a}}=1:$ $3: 7: 10$. Here, we take $\left[Y_{\tilde{a}}\right]_{t \ll \Gamma_{\tilde{a}}^{-1}}=Y_{\tilde{a}}^{(\mathrm{eq})}$. Numbers in the figure are the values of $\Omega_{\tilde{W}} h^{2}$.

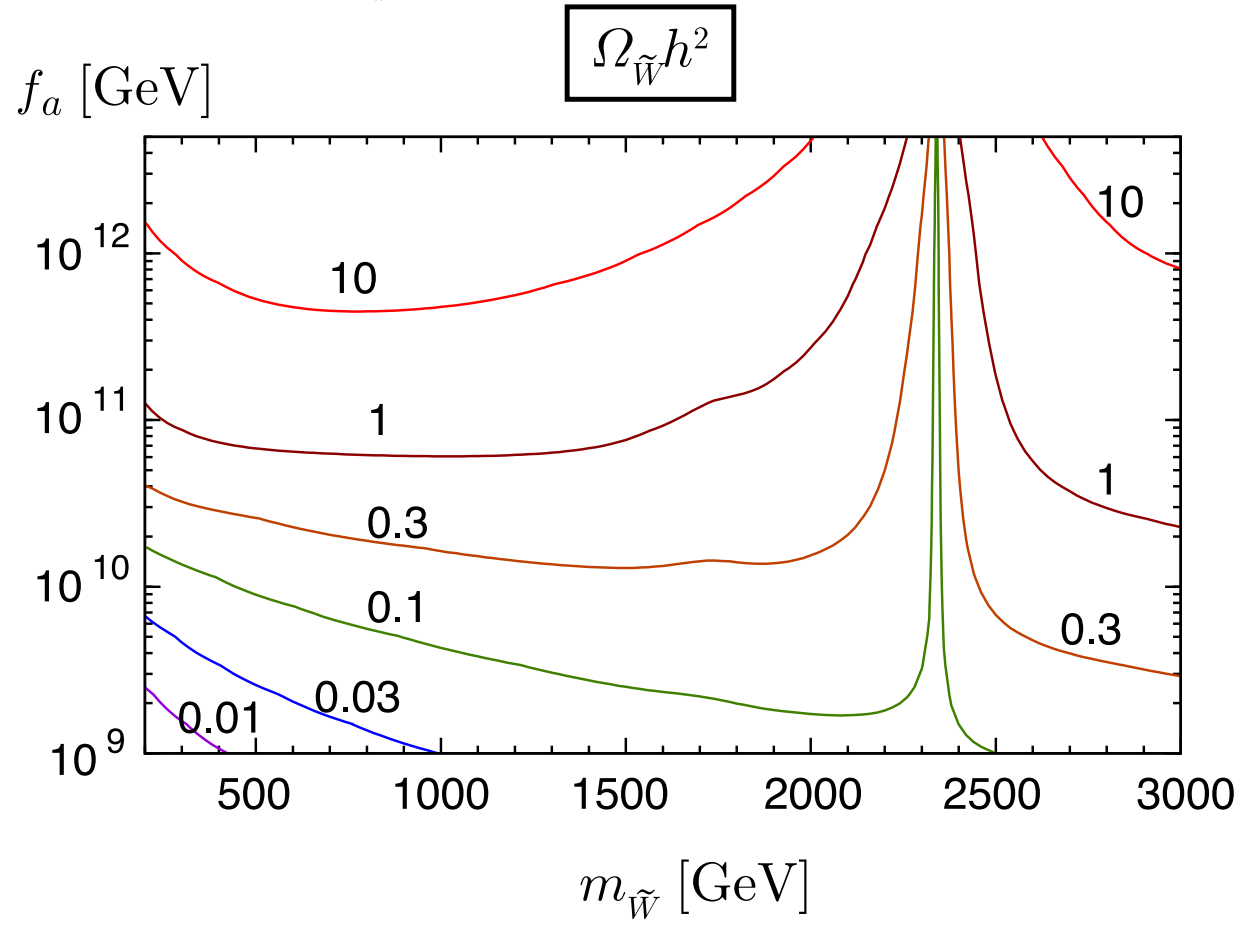

Figure 9: Same as Fig. 8, except for $m_{\tilde{W}}: m_{\tilde{B}}: m_{\tilde{g}}: m_{\tilde{a}}=1: 3: 7: 5$. 


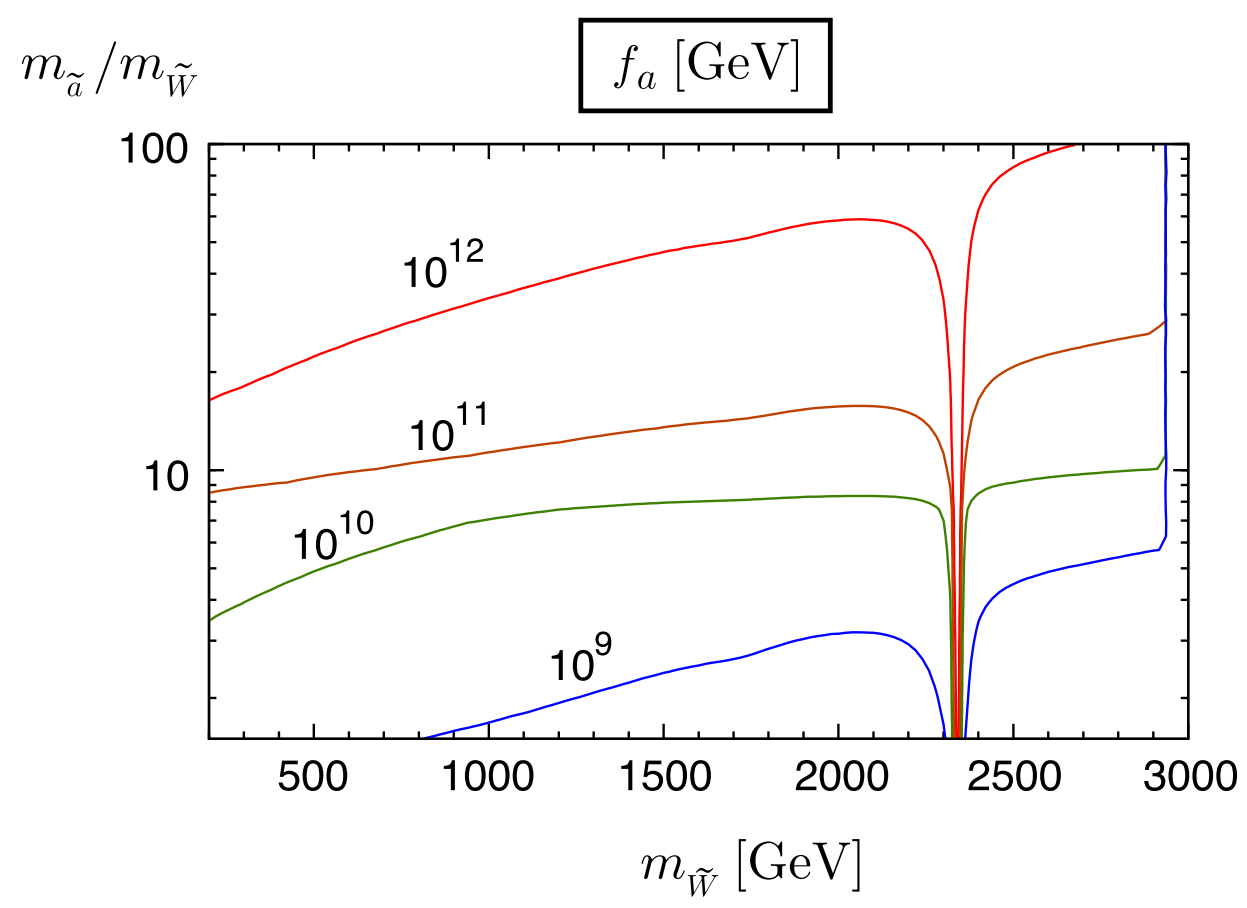

Figure 10: Contours of constant $f_{a}$ which gives $\Omega_{\tilde{W}}=\Omega_{c}$ for $\left[Y_{\tilde{a}}\right]_{t \ll \Gamma_{\tilde{a}}^{-1}}=Y_{\tilde{a}}^{(\mathrm{eq})}$. The horizontal axis is the Wino mass while the vertical one is the ratio $m_{\tilde{a}} / m_{\tilde{W}}$. The gaugino masses are assumed to obey the anomaly-mediation relation $m_{\tilde{W}}: m_{\tilde{B}}: m_{\tilde{g}}=1: 3: 7$.

$m_{\tilde{a}}=1: 3: 7: 5$. Then, the value of $f_{a}$ giving rise to $\Omega_{\tilde{W}}=\Omega_{c}$ is given by $1.5 \times 10^{10} \mathrm{GeV}$ $\left(1.0 \times 10^{10} \mathrm{GeV}, 5.1 \times 10^{9} \mathrm{GeV}, 2.2 \times 10^{9} \mathrm{GeV}\right)$ for $m_{\tilde{W}}=300 \mathrm{GeV}\left(m_{\tilde{W}}=500 \mathrm{GeV}\right.$, $m_{\tilde{W}}=1 \mathrm{TeV}$, and $\left.m_{\tilde{W}}=2 \mathrm{TeV}\right)$.

We have also studied how the required value of $f_{a}$ to realize the Wino dark matter depends on the axino mass. In Fig. 10, we show contours of constant $f_{a}$ which gives $\Omega_{\tilde{W}}=\Omega_{c}$ for $\left[Y_{\tilde{a}}\right]_{t \ll \Gamma_{\tilde{a}}^{-1}}=Y_{\tilde{a}}^{(\mathrm{eq})}$ on $m_{\tilde{W}}$ vs. $m_{\tilde{a}} / m_{\tilde{W}}$ plane.

So far, we have adopted the thermal abundance of axino. Even if the primordial abundance of axino is smaller, the resultant Wino density does not change as far as Winos produced by decay of axino are much enough to pair-annihilate. We also performed the calculation with lower reheating temperature, and checked that the results are more or less unchanged.

\section{Conclusions and Discussion}

In this paper, we have studied the possibility of the Wino cold dark matter in supersymmetric models, paying particular attentions to the scenario in which the decay of a long-lived particle $X$ produces significant amount of the Wino LSP. We have numerically calculated the relic abundance of the neutral Wino, carefully taking account of the effects of coannihilation and 
Sommerfeld effects. We have seen that the Sommerfeld effect drastically enhances the pair annihilation cross section of the Wino if $m_{\tilde{W}} \gtrsim 1 \mathrm{TeV}$. We have studied the cases where moduli fields, gravitino, or axino plays the role of $X$, and derived the constraints on the model parameters in each case.

So far, we have not discussed cosmological and astrophysical constraints on the Wino dark matter. Because the neutral Wino has large annihilation cross section, the Wino dark matter scenario conflicts with astrophysical and cosmological constraints if the Wino mass is too small. One of the constraints is from the negative observation of high energy $\gamma$-ray from dwarf galaxies, from which the mass regions $m_{\tilde{W}} \lesssim 400 \mathrm{GeV}$ are disfavored [51, 52] although astrophysical uncertainties exist. Another constraint is from BBN. The relic Wino may pair annihilated during and after the BBN epoch, which causes photo- and hadro-dissociation processes. In order not to spoil the success of the BBN scenario, the Wino mass is required to be larger than $\sim 200 \mathrm{GeV}[53$. Thus, these astrophysical and cosmological constraints do not exclude most of the parameter regions we have studied.

Acknowledgements: This work is supported in part by Grant-in-Aid for Scientific research from the Ministry of Education, Science, Sports, and Culture (MEXT), Japan, No. 22244021, No. 22540263, and No. 23104008.

\section{A Sommerfeld Enhancement Factors}

Here we present the possible decay modes and the decay widths for each set of $(Q, S)$, which are summarized in Table 1. The annihilation cross section to the $f f^{\prime}$ final state is obtained by

$$
\sigma v=c \frac{\pi^{2} \alpha_{2}^{2}}{m_{\tilde{W}}^{2}} \sum_{S=0,1}(2 S+1) P\left(x_{f}, x_{f^{\prime}}\right) D|A|^{2},
$$

where $c=2$ for the annihilation of identical particles, otherwise $c=1$. The dynamical factor $D$ and the Sommerfeld factor $|A|^{2}$ are given in Table 1.

The phase factor $P\left(x_{1}, x_{2}\right)$ is given by

$$
P\left(x_{1}, x_{2}\right)=\sqrt{1-\frac{x_{1}^{2}+x_{2}^{2}}{2}+\frac{\left(x_{1}^{2}-x_{2}^{2}\right)^{2}}{16}} .
$$




\begin{tabular}{|c|c|c|c|}
\hline initial state & final state & Dynamical factor $(D)$ & Sommerfeld factor $\left(|A|^{2}\right)$ \\
\hline$\chi^{0} \chi^{0}(S=0)$ & $W^{+} W^{-}$ & $\frac{1}{2}\left[1-x_{W}^{2}\right]\left[1-\frac{x_{W}^{2}}{2}\right]^{-2}$ & $\left|A_{21}^{(0,0)}+\sqrt{2} A_{22}^{(0,0)}\right|^{2}$ \\
& $Z Z$ & $c_{W}^{4}\left[1-x_{Z}^{2}\right]\left[1-\frac{x_{Z}^{2}}{2}\right]^{-2}$ & $\left|A_{21}^{(0,0)}\right|^{2}$ \\
& $Z \gamma$ & $2 c_{W}^{2} s_{W}^{2}$ & $\left|A_{21}^{(0,0)}\right|^{2}$ \\
& $\gamma \gamma$ & $s_{W}^{4}$ & $\left|A_{21}^{(0,0)}\right|^{2}$ \\
\hline$\chi^{+} \chi^{-}(S=0)$ & $W^{+} W^{-}$ & $\frac{1}{2}\left[1-x_{W}^{2}\right]\left[1-\frac{x_{W}^{2}}{2}\right]^{-2}$ & $\left|A_{11}^{(0,0)}+\sqrt{2} A_{12}^{(0,0)}\right|^{2}$ \\
& $Z Z$ & $c_{W}^{4}\left[1-x_{Z}^{2}\right]\left[1-\frac{x_{Z}^{2}}{2}\right]^{-2}$ & $\left|A_{11}^{(0,0)}\right|^{2}$ \\
& $Z \gamma$ & $2 c_{W}^{2} s_{W}^{2}$ & $\left|A_{11}^{(0,0)}\right|^{2}$ \\
& $\gamma \gamma$ & $s_{W}^{4}$ & $\left|A_{11}^{(0,0)}\right|^{2}$ \\
\hline$\chi^{+} \chi^{-}(S=1)$ & $W^{+} W^{-}$ & $\frac{1}{48} f_{1}\left(x_{W}, x_{Z}\right)$ & $\left|A^{(0,1)}\right|^{2}$ \\
& $Z h$ & $\frac{1}{48} f_{3}\left(x_{Z}, x_{h}\right)$ & $\left|A^{(0,1)}\right|^{2}$ \\
\hline$\chi^{+} \chi^{0}(S=0)$ & $W^{+} Z$ & $\frac{N_{W}^{2}}{2} f_{6}\left(x_{W}, x_{Z}\right)$ & $\left|A^{(1,0)}\right|^{2}$ \\
& $W^{+} \gamma$ & $\frac{s_{W}^{2}}{2}$ & $\left|A^{(1,0)}\right|^{2}$ \\
\hline$\chi^{+} \chi^{0}(S=1)$ & $W^{+} Z$ & $\frac{1}{48} f_{2}\left(x_{W}, x_{Z}\right)$ & $\left|A^{(1,1)}\right|^{2}$ \\
& $W^{+} h$ & $\frac{1}{48} f_{3}\left(x_{W}, x_{h}\right)$ & $\left|A^{(1,1)}\right|^{2}$ \\
& $u \bar{d}$ & $\frac{1}{4} f_{5}\left(x_{W}, x_{d}, x_{u}\right)$ & $\frac{1}{12} f_{5}\left(x_{W}, x_{e}, 0\right)$ \\
\hline$\chi^{+} \chi^{+}(S=0)$ & $W^{+} W^{+}$ & $\frac{1}{2}\left[1-x_{W}^{2}\right]\left[1-\frac{x_{W}^{2}}{2}\right]^{-2}$ & $\left|x^{2}\right|^{2}$ \\
\hline
\end{tabular}

Table 1: Table of parameters to calculate annihilation cross sections for each initial and final states. Tree-level cross sections are recovered by setting $A_{11}^{(0,0)}=A_{22}^{(0,0)}=A^{(0,1)}=A^{(1,0)}=$ $A^{(1,1)}=A^{(2,0)}=1$ and $A_{21}^{(0,0)}=A_{12}^{(0,0)}=0$. Here, $x_{I}$ is the mass of the particle $I$ normalized by $m_{\tilde{W}}, x_{I} \equiv m_{I} / m_{\tilde{W}}$. $f$ denotes quarks and leptons. $N_{C}$ is the color factor; $N_{C}=3$ (1) for quarks (leptons). For the calculation of the Sommerfeld factors $A^{(Q, S)}$ for states with charge $Q$ and spin $S$, see [30]. 
In addition, the functions $f_{i}(x)$ are defined as

$$
\begin{aligned}
f_{1}\left(x_{W}, x_{Z}\right) & =\frac{4\left(1-x_{W}^{2}\right)\left(4+20 x_{W}^{2}+3 x_{W}^{4}\right)\left(2+x_{W}^{2}-x_{Z}^{2}\right)^{2}}{\left(2-x_{W}^{2}\right)^{2}\left(4-x_{Z}^{2}\right)^{2}}, \\
f_{2}\left(x_{W}, x_{Z}\right) & =\frac{\left\{\left(4-x_{W}^{2}-x_{Z}^{2}\right)^{2}-4 x_{W}^{2} x_{Z}^{2}\right\}\left(16+40 x_{W}^{2}+40 x_{Z}^{2}+10 x_{W}^{2} x_{Z}^{2}+x_{W}^{4}+x_{Z}^{4}\right)}{\left(4-x_{W}^{2}\right)^{2}\left(4-x_{W}^{2}-x_{Z}^{2}\right)^{2}}, \\
f_{3}\left(x, x_{h}\right) & =\left(1-\frac{x^{2}}{4}\right)^{-2}\left(1+\frac{5 x^{2}-x_{h}^{2}}{2}+\frac{\left(x^{2}-x_{h}^{2}\right)^{2}}{16}\right) \\
f_{4}\left(x_{Z}, x_{f}\right) & =\left(1-\frac{x_{Z}^{2}}{4}\right)^{-2}\left[\left(1-c_{f} x_{Z}^{2}+\frac{c_{f}^{2}}{2} x_{Z}^{4}\right)-\frac{x_{f}^{2}}{4}\left(1+2 c_{f} x_{Z}^{2}-c_{f}^{2} x_{Z}^{4}\right)\right] \\
f_{5}\left(x_{W}, x_{d}, x_{u}\right) & =\left(1-\frac{x_{W}^{2}}{4}\right)^{-2}\left(1-\frac{x_{u}^{2}+x_{d}^{2}}{8}-\frac{\left(x_{u}^{2}-x_{d}^{2}\right)^{2}}{32}\right) \\
f_{6}\left(x_{W}, x_{Z}\right) & =\left(1-\frac{x_{W}^{2}+x_{Z}^{2}}{4}\right)^{-2}\left(1-\frac{x_{W}^{2}+x_{Z}^{2}}{2}+\frac{\left(x_{W}^{2}-x_{Z}^{2}\right)^{2}}{16}\right),
\end{aligned}
$$

where $c_{f}=Q_{f} s_{W}^{2} /\left(2 T_{3 L}^{f}\right)$ for the particle with the electric charge $Q_{f}$ and the weak isospin $T_{3 L}^{f}$.

\section{References}

[1] G. D. Coughlan, W. Fischler, E. W. Kolb, S. Raby and G. G. Ross, Phys. Lett. B 131 (1983) 59.

[2] J. R. Ellis, D. V. Nanopoulos and M. Quiros, Phys. Lett. B 174 (1986) 176.

[3] A. S. Goncharov, A. D. Linde and M. I. Vysotsky, Phys. Lett. B 147 (1984) 279.

[4] T. Banks, D. B. Kaplan and A. E. Nelson, Phys. Rev. D 49 (1994) 779.

[5] B. de Carlos, J. A. Casas, F. Quevedo and E. Roulet, Phys. Lett. B 318 (1993) 447.

[6] M. Kawasaki, K. Kohri and T. Moroi, Phys. Lett. B 625 (2005) 7.

[7] M. Kawasaki, K. Kohri and T. Moroi, Phys. Rev. D 71 (2005) 083502.

[8] G. F. Giudice, M. A. Luty, H. Murayama and R. Rattazzi, JHEP 9812 (1998) 027.

[9] L. Randall and R. Sundrum, Nucl. Phys. B 557 (1999) 79.

[10] T. Yanagida, Phys. Lett. B 344 (1995) 211.

[11] G. Aad et al. [ATLAS Collaboration], Phys. Lett. B 716 (2012) 1.

[12] S. Chatrchyan et al. [CMS Collaboration], Phys. Lett. B 716 (2012) 30. 
[13] See, for example, G. F. Giudice and A. Strumia, Nucl. Phys. B 858 (2012) 63.

[14] T. Moroi and L. Randall, Nucl. Phys. B 570 (2000) 455.

[15] B. S. Acharya, K. Bobkov, G. L. Kane, J. Shao and P. Kumar, Phys. Rev. D 78 (2008) 065038 .

[16] B. S. Acharya, P. Kumar, K. Bobkov, G. Kane, J. Shao and S. Watson, JHEP 0806 (2008) 064.

[17] H. Baer, A. Lessa, S. Rajagopalan and W. Sreethawong, JCAP 1106 (2011) 031.

[18] H. Baer, A. Lessa and W. Sreethawong, JCAP 1201 (2012) 036.

[19] T. Moroi and K. Nakayama, Phys. Lett. B 710 (2012) 159.

[20] G. B. Gelmini and P. Gondolo, Phys. Rev. D 74 (2006) 023510.

[21] G. Gelmini, P. Gondolo, A. Soldatenko and C. E. Yaguna, Phys. Rev. D 74 (2006) 083514.

[22] M. Nagai and K. Nakayama, Phys. Rev. D 76 (2007) 123501.

[23] M. Nagai and K. Nakayama, Phys. Rev. D 78 (2008) 063540.

[24] J. L. Feng, T. Moroi, L. Randall, M. Strassler and S. -f. Su, Phys. Rev. Lett. 83 (1999) 1731.

[25] M. Ibe, S. Matsumoto and R. Sato, arXiv:1212.5989 [hep-ph].

[26] J. Hisano, K. Kohri and M. M. Nojiri, Phys. Lett. B 505 (2001) 169.

[27] M. Ibe, A. Kamada and S. Matsumoto, arXiv:1210.0191 [hep-ph].

[28] J. Hisano, S. .Matsumoto, M. M. Nojiri and O. Saito, Phys. Rev. D 71 (2005) 063528.

[29] J. Hisano, S. Matsumoto, O. Saito and M. Senami, Phys. Rev. D 73 (2006) 055004.

[30] J. Hisano, S. Matsumoto, M. Nagai, O. Saito and M. Senami, Phys. Lett. B 646 (2007) 34 .

[31] G. Hinshaw et al., arXiv:1212.5226 [astro-ph.CO].

[32] M. Kawasaki, K. Kohri, T. Moroi and A. Yotsuyanagi, Phys. Rev. D 78 (2008) 065011.

[33] M. Endo, K. Hamaguchi and F. Takahashi, Phys. Rev. Lett. 96 (2006) 211301.

[34] S. Nakamura and M. Yamaguchi, Phys. Lett. B 638 (2006) 389. 
[35] M. Kawasaki, F. Takahashi and T. T. Yanagida, Phys. Lett. B 638 (2006) 8.

[36] M. Fukugita and T. Yanagida, Phys. Lett. B 174 (1986) 45.

[37] W. Buchmuller, P. Di Bari and M. Plumacher, Annals Phys. 315 (2005) 305.

[38] G. F. Giudice, A. Notari, M. Raidal, A. Riotto and A. Strumia, Nucl. Phys. B 685 (2004) 89.

[39] R. D. Peccei and H. R. Quinn, Phys. Rev. Lett. 38 (1977) 1440.

[40] R. D. Peccei and H. R. Quinn, Phys. Rev. D 16 (1977) 1791.

[41] S. Kim, W. -I. Park and E. D. Stewart, JHEP 0901 (2009) 015.

[42] M. Kawasaki, N. Kitajima and K. Nakayama, Phys. Rev. D 82 (2010) 123531.

[43] M. Kawasaki, N. Kitajima and K. Nakayama, Phys. Rev. D 83 (2011) 123521.

[44] T. Moroi and M. Takimoto, Phys. Lett. B 718 (2012) 105.

[45] K. Mukaida and K. Nakayama, JCAP 1301 (2013) 017.

[46] L. Covi, H. -B. Kim, J. E. Kim and L. Roszkowski, JHEP 0105 (2001) 033.

[47] A. Brandenburg and F. D. Steffen, JCAP 0408 (2004) 008.

[48] K. J. Bae, K. Choi and S. H. Im, JHEP 1108 (2011) 065.

[49] K. -Y. Choi, L. Covi, J. E. Kim and L. Roszkowski, JHEP 1204 (2012) 106.

[50] K. -Y. Choi, J. E. Kim, H. M. Lee and O. Seto, Phys. Rev. D 77 (2008) 123501.

[51] M. Ackermann et al. [Fermi-LAT Collaboration], Phys. Rev. Lett. 107 (2011) 241302.

[52] K. N. Abazajian and J. P. Harding, JCAP 1201 (2012) 041.

[53] J. Hisano, M. Kawasaki, K. Kohri and K. Nakayama, Phys. Rev. D 79 (2009) 063514 [Erratum-ibid. D 80 (2009) 029907]. 